\title{
Características do ambiente do bairro e prática de caminhada no lazer e deslocamento em idosos
}

\author{
Characteristics of the neighborhood environment and commuting and \\ leisure walking in elderly
}

Giovane Pereira Balbé1, Clair Antônio Wathier', Cassiano Ricardo Rech²

\section{Resumo}

Este estudo teve como objetivo analisar a prevalência e as características do ambiente do bairro associadas com a caminhada no lazer e no deslocamento em idosos. Estudo transversal com 213 idosos (60 a 93 anos; 78,9\% mulheres), participantes de grupos de convivência da zona urbana de Rio do Sul (SC), no ano de 2014. A caminhada no lazer e deslocamento foi avaliada utilizando a versão longa do Questionário Internacional de Atividade Física (ativo $\geq 150$ minutos/sem). A percepção do ambiente foi mensurada a partir da versão modificada da Neighborhood Environmental Walkability Scale, composta de 13 questões sobre as características do ambiente do bairro. Adotaram-se análises de regressão logística binária bruta e ajustada, com nível de significância de 5\%. A prevalência de caminhada no lazer foi de 22,1\% (IC95\%: 16,4-27,7) e de 28,2\% (IC95\%: 22,1-34,2) no deslocamento. A percepção de existência de ruas inclinadas $(\mathrm{OR}=3,18$; IC95\%: 1,44-7,05) e de academias ao ar livre (OR= 0,16; IC95\%: 0,04-0,70) foi associada com caminhada no lazer. No deslocamento, observou-se que maior chance de ser ativo na caminhada entre idosos que perceberam à presença de calçadas em condições de uso $(\mathrm{OR}=$ 3,50; IC95\%: 1,48-8,28) e a presença de academias de ginástica ( $\mathrm{OR}=4,16$; IC95\%: 1,72-12,3). Conclui-se que menos de $30 \%$ dos idosos de Rio do Sul são ativos na caminhada no lazer e no deslocamento e, a presença de calçadas em condições adequadas de uso foi associada com o comportamento ativo na caminhada no deslocamento em idosos. Melhorar a estrutura das calçadas e diversificar os espaços para atividade física pode promover maiores níveis de caminhada em idosos.

\section{Palavras-chave}

Ambiente construído; Atividade motora; Planejamento urbano; Envelhecimento.

\begin{abstract}
This study aimed to analyze the prevalence and characteristics of the neighborhood environment associated of walking in leisure and commuting in older adults. Cross-sectional study of 213 older adults (60 to 93 years; 78,9\% women), participants in urban area city of Rio do Sul, SC, in 2014. The walking in leisure and commuting was assessed using International Physical Activity Questionnaire (active $\geq 150$ minutes/week). The perception of the environment was measured from the modified version of the Neighborhood Environment Walkability Scale, composed of 13 questions about the characteristics of the neighborhood environment. They were adopted binary logistic regression analyses, with 5\% significance level. The prevalence of walking during leisure time was 22.1\% (CI95\%: 16.4-27.7) and 28.2\% (CI95\%: 22.1-34.2) in the commuting. The perception of climbs streets $(O R=3.18$; C195\%: 1.44-7.05) and of outdoor gyms (OR= 0.16; CI95\%: 0.04-0.70) was associated with to be active in walking in leisure-time. In commuting was observed that greater chance of active in walking among older adults people who perceived the presence of sidewalks $(\mathrm{OR}=3.50$; CI95\%: 1.48 $8.28)$ and the presence of gyms $(O R=4.16$; $C I 95 \%: 1.72-12.3)$. It is concluded that less than $30 \%$ older adults of the Rio do Sul are active on walk in leisure and commuting, and the presence of sidewalks in poor conditions of use was associated with higher walks in the commuting in older adults. Thus, improving the structure of sidewalks and diversify the spaces for physical activity to structures can promote higher levels of walking in older adults.
\end{abstract}

\section{Keywords}

Built Environment; Motor Activity; Urban Planning; Aging.

\section{Introdução}

Intervenções focadas em estratégias apenas no su- jeito, sem considerar o contexto do ambiente que o mesmo está inserido, têm demonstrado pouco efeito, especialmente na manutenção da Atividade Física $(\mathrm{AF})^{1,2}$. Considerando a influência de variáveis de 
múltiplos níveis de mudança é plausível atentar para a importância do papel do ambiente social e físico nos baixos níveis de AF na população ${ }^{2-4}$. Desse modo, é crescente o interesse de discutir o papel das características do ambiente na caminhada em idosos 5 . A caminhada é AF mais praticada entre idosos, sendo de baixo impacto, com reduzido risco de lesão e de fácil acesso ${ }^{6-8}$. Além disso, a caminhada, tanto no lazer quanto no deslocamento está correlacionada ao menor risco de mortalidade por todas as causas 9 .

Estudos indicam associação entre características do ambiente e caminhada no lazer e deslocamento em idosos ${ }^{8,10,11}$. A maior parte destes achados referem-se a grandes centros urbanos, principalmente em se tratando de pesquisas conduzidas no Brasil ${ }^{12,13-16}$. Nesse cenário, pouco se conhece sobre esse contexto em cidades menores que envolvem características ambientais como à disponibilidade de locais para $\mathrm{AF}$, à presença de calçadas, à segurança e à estética. Acredita-se que diferenças quanto ao menor tráfego, menor taxa de crimes, menores distâncias até os locais de compras, lazer e trabalho, possam estimular a prática de caminhada em centros urbanos menores. Consequentemente, os idosos podem perceber de modo diferente as características do ambiente nesses locais para caminhada quando comparado com idosos que vivem em grandes cidades ${ }^{8}$.

Desta forma, investigar a relação das características do ambiente do bairro com a caminhada em idosos, de uma cidade de pequeno porte, pode contribuir com novas evidências quanto aos aspectos ambientais relacionados ao comportamento ativo dessa população. Com isso, esse estudo tem como objetivo analisar a prevalência e as características do ambiente do bairro associadas com a caminhada no lazer e no deslocamento em idosos de Rio do Sul, Santa Catarina.

\section{Métodos}

Estudo transversal realizado com uma amostra de idosos ( $\geq 60$ anos), de ambos os sexos, participantes de grupos de convivência na zona urbana de Rio do Sul, Santa Catarina, conduzido entre Julho e Outubro de 2014. Destaca-se os grupos de convivência por serem locais situados em diferentes bairros, e que geralmente, congregam grande número de idosos o que possibilitou a realização deste estudo.

O município de Rio do Sul está localizado no Alto Vale do Itajaí (Região Nordeste), em Santa Catarina, com uma área de $260,357 \mathrm{~km}^{2}$ e população aproximada de 61.198 habitantes, sendo 6.090 idosos residentes na zona urbana ${ }^{17}$. O Índice de Desenvolvimento Humano (IDH) municipal é de 0,802 , o que o coloca em $36^{\text {a }}$ posição entre os municípios brasileiros com maior $\operatorname{IDH}^{18}$ e, é considerado elevado.

O população do estudo foi composta por todos os idosos de Rio do Sul que frequentavam grupos de convivência. No total 300 idosos estavam cadastrados nos grupos em 2014. Todos os grupos foram visitados e os idosos convidados a participar do estudo. Foram considerados inelegíveis os idosos com problemas físicos que limitavam o deslocamento na forma de caminhada e aqueles ausentes nos dias das visitas, após três tentativas. O tamanho amostral permite estimar associações com um poder $\geq 0,80$ considerando estimativas de associação de 1,80 e uma estimativa de $35 \%$ nos desfechos ${ }^{16}$. As estimativas do poder da amostra foram realizadas a posteriori por meio do software Gpower 3.1. ${ }^{19}$

A coleta de dados foi conduzida no período de julho a outubro de 2014, por entrevistadores treinados, com ensino superior completo ou em fase de conclusão de curso em Educação Física. Foi aplicado questionário estruturado com questões pré-codificadas na forma de entrevista face a face. Anteriormente a coleta ocorreu o treinamento dos entrevistadores, para a familiarização com o questionário 
e padronização das condutas de campo. Os instrumentos foram testados em um grupo de idosos para analisar a adequação quanto ao tempo de entrevista e procedimentos de coleta.

O desfecho deste estudo refere-se à caminhada no lazer e no deslocamento, mensurada por meio do Questionário Internacional de Atividade Física (IPAQ), versão longa, adaptado e validado para idosos no Brasil, com validade concorrente de 0,54 e concordância intraclasse de $0,88^{20}$. A caminhada no lazer e no deslocamento foi obtida pela multiplicação, considerando uma semana normal, da frequência semanal de caminhada pelo tempo mínimo de 10 minutos contínuos ou mais por semana. A partir disso, calculou-se o tempo de caminhada para cada domínio (lazer, deslocamento) de forma individual, o qual foi dicotomizado em ativos $\geq 150$ minutos por semana e insuficientemente ativos $\leq 149$ minutos por semana ${ }^{6}$.

A variável exposição foi à percepção do ambiente do bairro, avaliada pela escala Neighborhood Environmental Walkability Scale (A-NEWS), traduzida e validada para a realidade brasileira, com reprodutibilidade de 0,80 e concordância intraclasse de $0,98^{21}$. Para o presente estudo, foram selecionadas 13 questões, relacionadas à: estruturas físicas próximas da residência (seis questões); calçadas (uma questão); estética do bairro (uma questão); segurança no trânsito (duas questões); segurança geral (duas questões); e topografia das ruas (uma questão). Os idosos foram orientados para considerar como locais próximos de sua residência àqueles que pudessem chegar caminhando em até 10 minutos. As questões da escala A-NEWS foram compostas por opções de respostas categorizadas de forma dicotômica (sim ou não).

Considerou-se como variáveis de caracterização da amostra sexo (masculino, feminino), faixa etária em anos (60 a 69, 70 a 79, 80 ou mais), escolaridade em anos de estudo ( 0 a 4, 5 a 8, $\geq 9$ ), estado civil (solteiro/viúvo/divorciado, casado), renda média familiar em salários mínimos $(<1,1$ a $2, \geq 3$ ), arranjo familiar (sozinho, cônjuge/familiares) e estado de saúde (ótima/boa, regular/ruim), obtida por meio da pergunta "Como você avalia sua condição de saúde?". Estas variáveis foram coletadas mediante questionário elaborado pelos pesquisadores para identificação das características da amostra.

Todas as variáveis do estudo foram analisadas de forma descritiva por meio de frequência absoluta e relativa. Utilizou-se o teste de qui-quadrado para investigar possíveis diferenças entre os sexos e as variáveis de caracterização da amostra e dos desfechos. Para verificar a associação entre a caminhada de lazer e descolamento (variável dependente) com as variáveis ambientais (independentes) utilizou-se a regressão logística binária bivariada e multivariada, com intervalo de confiança de $95 \%$. Após a regressão bivariada todas as variáveis foram ajustadas com a entrada forçada de todas as variáveis independentes no mesmo modelo. As variáveis de sexo, faixa etária, escolaridade, estado civil, renda média familiar e estado de saúde foram utilizadas como ajuste e adicionadas ao modelo final. Os dados foram tabulados e analisados pelo programa Stata 12.0.

O estudo foi aprovado no Comitê de Ética em Pesquisa da Universidade do Vale do Itajaí (parecer $\mathrm{n}^{\circ}$ 685.806). Todos os participantes foram voluntários e assinaram o Termo de Consentimento Livre e Esclarecido (resolução 446/2012).

\section{Resultados}

O número de idosos cadastrados nos grupos de convivência em Rio do Sul foi de 300 indivíduos, destes, participaram do estudo 213 idosos (78,9\% mulheres) entre 60 a 93 anos de idade. Houveram 27 idosos considerados não elegíveis por 
apresentar limitações físicas para caminhada e 60 idosos que estavam ausentes em pelo menos três dias de visitas realizadas.

A amostra foi composta predominantemente por idosos entre 60 e 69 anos $(52,6 \%)$, casados $(54,5 \%)$, com até quatro anos de estudo $(56,8 \%)$, com renda entre 1 a 2 salários mínimos $(53,1 \%)$, aposentados $(74,6 \%)$ e que residem com o cônjuge ou familiares $(77,9 \%)$. Na Tabela 1 , observa-se uma maior proporção de mulheres solteiras e que moram sozinha $(51,8 \%$; $p<0,001$ e $26,8 \%$; $p=0,003$, respectivamente), quando comparadas aos homens. Por outro lado, diferenças significativas ocorreram nos homens com nove anos ou mais de estudo $(31,1 \%$; $=0,023)$ e com renda média familiar de três ou mais salários mínimos $(53,3 \%$; $\mathrm{p}=0,035)$.

TABELA 1 - Características da amostra de idosos, Rio do Sul, SC, $2014(n=213)$.

\begin{tabular}{|c|c|c|c|c|c|c|c|}
\hline \multirow{2}{*}{ Variáveis } & \multicolumn{2}{|c|}{$\begin{array}{c}\text { Total } \\
(n=213)\end{array}$} & \multicolumn{2}{|c|}{$\begin{array}{c}\text { Homens } \\
(n=45 ; 21,1 \%)\end{array}$} & \multicolumn{2}{|c|}{$\begin{array}{c}\text { Mulheres } \\
(n=168 ; 78,9 \%)\end{array}$} & \multirow[t]{2}{*}{$\mathrm{p}$} \\
\hline & $n$ & $\%$ & $n$ & $\%$ & $n$ & $\%$ & \\
\hline \multicolumn{8}{|l|}{ Faixa etária (anos) } \\
\hline 60 a 69 & 112 & 52,6 & 23 & 51,1 & 89 & 53,0 & \multirow[t]{3}{*}{0,844} \\
\hline 70 a 79 & 82 & 38,5 & 17 & 37,8 & 65 & 38,7 & \\
\hline 80 ou mais & 19 & 8,9 & 5 & 11,1 & 14 & 8,3 & \\
\hline \multicolumn{8}{|l|}{ Estado civil } \\
\hline Solteiro, viúvo, divorciado & 97 & 45,5 & 10 & 22,2 & 87 & 51,8 & \multirow[t]{2}{*}{$<0,001$} \\
\hline Casado & 116 & 54,5 & 35 & 77,8 & 81 & 48,2 & \\
\hline \multicolumn{8}{|l|}{ Escolaridade (anos de estudo) } \\
\hline 0 a 4 & 121 & 56,8 & 22 & 48,9 & 99 & 58,9 & \multirow[t]{3}{*}{0,023} \\
\hline 5 a 8 & 55 & 25,8 & 9 & 20,0 & 46 & 27,4 & \\
\hline$\geq 9$ & 37 & 17,4 & 14 & 31,1 & 23 & 13,7 & \\
\hline \multicolumn{8}{|l|}{ Renda média familiar* } \\
\hline$<1$ & 16 & 7,5 & 2 & 4,4 & 14 & 8,3 & \multirow[t]{3}{*}{0,035} \\
\hline $1 \mathrm{a} 2$ & 113 & 53,1 & 19 & 42,2 & 94 & 56,0 & \\
\hline$\geq 3$ & 84 & 39,4 & 24 & 53,3 & 60 & 35,7 & \\
\hline \multicolumn{8}{|l|}{ Arranjo familiar } \\
\hline Sozinho & 47 & 22,1 & 2 & 4,4 & 45 & 26,8 & \multirow[t]{2}{*}{0,001} \\
\hline Cônjuge ou familiares & 166 & 77,9 & 43 & 95,6 & 123 & 73,2 & \\
\hline \multicolumn{8}{|l|}{ Caminhada no lazer } \\
\hline Insuf. ativos ( $\leq 149$ minutos/semana) & 166 & 77,9 & 31 & 68,9 & 135 & 80,4 & \multirow[t]{2}{*}{0,099} \\
\hline Ativos ( $\geq 150$ minutos/semana) & 47 & 22,1 & 14 & 31,1 & 33 & 19,6 & \\
\hline \multicolumn{8}{|l|}{ Caminhada no deslocamento } \\
\hline Insuf. ativos ( $\leq 149$ minutos/semana) & 153 & 71,8 & 30 & 66,7 & 123 & 73,2 & \multirow{2}{*}{0,386} \\
\hline Ativos ( $\geq 150$ minutos/semana) & 60 & 28,2 & 15 & 33,3 & 45 & 26,8 & \\
\hline
\end{tabular}

* salário mínimo (R\$ 724,00); Insuf. ativos= Insuficientemente ativos; Teste de qui-quadrado.

A prevalência de idosos que cumpriram com a recomendação de caminhada no lazer ( $\geq 150$ minutos por semana) foi de $22,1 \%$ (IC95\%: 16,4-27,7), sendo maior entre os homens $(31,1 \%$ versus $19,6 \%$; $\mathrm{p}=0,099)$, porém, sem diferença significativa. Já a proporção de idosos que caminham 150 minutos ou mais por semana no deslocamento foi de 28,2\% (IC95\%: 22,1-34,2) (33,3\% homens versus $26,8 \%$ mulheres, $\mathrm{p}=0,386$ ).

A Tabela 2 apresenta a associação bruta e ajustada entre as características do ambiente do bairro e a caminhada de lazer. Nenhuma característica do ambiente 
do bairro foi associada com o desfecho na análise bruta. Porém, na análise ajustada, observou-se academias ao ar livre ( $\mathrm{OR}=0,16$; IC95\%: 0,04-0,70) e existência de ruas inclinadas $(\mathrm{OR}=3,18$; IC95\%: 1,44-7,05) associadas a caminhada no lazer, no entanto, a presença de academias ao ar livre indica uma menor chance de idosos cumprirem as recomendações de caminhada nesse domínio.

TABELA 2 - Associação bruta e ajustada entre os fatores ambientais do bairro e caminhada no lazer ( $\geq 150$ min./sem.) em idosos de Rio do Sul (SC) 2014 ( $n=213)$.

\begin{tabular}{|c|c|c|c|c|c|c|c|}
\hline \multirow{3}{*}{ Variáveis } & & \multicolumn{6}{|c|}{ Caminhada no lazer ( $\geq 150 \mathrm{~min} . / \mathrm{sem})}$. \\
\hline & & \multirow{2}{*}{$n$} & \multirow{2}{*}{$\%$} & \multicolumn{2}{|c|}{ Bruta } & \multicolumn{2}{|c|}{ Ajustada $^{a}$} \\
\hline & & & & OR & IC95\% & OR & IC95\% \\
\hline \multirow[t]{2}{*}{ Presença de academias de ginástica } & Não & 11 & 22,0 & 1 & & 1 & \\
\hline & Sim & 36 & 22,1 & 1,00 & $0,47-2,15$ & 0,79 & $0,30-2,06$ \\
\hline \multirow[t]{2}{*}{ Presença de parques } & Não & 3 & 11,1 & 1 & & 1 & \\
\hline & Sim & 44 & 23,7 & 2,48 & $0,71-8,62$ & 2,26 & $0,39-1,29$ \\
\hline \multirow[t]{2}{*}{ Presença de praças } & Não & 6 & 14,6 & 1 & & 1 & \\
\hline & Sim & 41 & 23,8 & 1,82 & $0,71-4,64$ & 3,72 & $0,60-2,31$ \\
\hline \multirow[t]{2}{*}{ Presença de centro comunitário } & Não & 12 & 22,2 & 1 & & 1 & \\
\hline & Sim & 35 & 22,0 & 0,98 & $0,46-2,07$ & 1,19 & $0,45-3,12$ \\
\hline \multirow[t]{2}{*}{ Presença de academia ao ar livre } & Não & 9 & 25,7 & 1 & & 1 & \\
\hline & Sim & 38 & 21,3 & 0,78 & $0,34-1,81$ & 0,16 & $0,04-0,70$ \\
\hline \multirow[t]{2}{*}{ Presença de igrejas, templos, cultos } & Não & 23 & 19,5 & 1 & & 1 & \\
\hline & Sim & 24 & 25,3 & 1,39 & $0,72-2,67$ & 1,62 & $0,68-3,87$ \\
\hline \multirow[t]{2}{*}{ Presença de calçadas } & Não & 22 & 25,0 & 1 & & 1 & \\
\hline & Sim & 25 & 20,0 & 0,75 & $0,39-1,43$ & 1,19 & $0,49-2,88$ \\
\hline \multirow[t]{2}{*}{ Presença de árvores } & Não & 29 & 20,7 & 1 & & 1 & \\
\hline & Sim & 18 & 24,7 & 1,25 & $0,64-2,45$ & 1,25 & $0,56-2,77$ \\
\hline \multirow[t]{2}{*}{ Trânsito intenso no bairro } & Não & 22 & 20,4 & 1 & & 1 & \\
\hline & Sim & 25 & 23,8 & 1,22 & $0,64-2,33$ & 1,47 & $0,66-3,28$ \\
\hline \multirow[t]{2}{*}{ Existem faixas, sinais e passarelas } & Não & 31 & 24,8 & 1 & & 1 & \\
\hline & Sim & 16 & 18,2 & 0,67 & $0,34-1,32$ & 1,47 & $0,66-3,28$ \\
\hline \multirow[t]{2}{*}{ Existem muitos crimes no bairro } & Não & 42 & 23,9 & 1 & & 1 & \\
\hline & Sim & 5 & 13,5 & 0,49 & $0,18-1,36$ & 0,40 & $0,13-1,22$ \\
\hline \multirow[t]{2}{*}{ É seguro caminhar durante a noite } & Não & 19 & 26,8 & 1 & & 1 & \\
\hline & Sim & 28 & 19,7 & 0,67 & $0,34-1,31$ & 0,57 & $0,22-1,43$ \\
\hline \multirow[t]{2}{*}{ Existência de ruas inclinadas } & Não & 16 & 14,3 & 1 & & 1 & \\
\hline & Sim & 31 & 30,7 & 2,65 & $1,34-5,23$ & 3,18 & $1,44-7,05$ \\
\hline
\end{tabular}

a Ajustada para sexo, faixa etária, escolaridade, estado civil, renda média familiar, estado de saúde e todas as variáveis independentes.

Quanto ao deslocamento (Tabela 3), a presença de calçadas (OR=3,50; IC95\%: $1,48-8,28)$ foi associada na análise bruta. Após ajuste para variáveis de confundimento, a presença de academias de ginástica (OR=4,16; IC95\%: 1,72-12,3) foi associada a caminhada no deslocamento, sendo que a existência de calçadas $(\mathrm{OR}=$ 1,96; IC95\%: 1,04-3,72) se manteve associada. 
TABELA 3 - Associação bruta e ajustada entre os fatores ambientais do bairro e caminhada no deslocamento ( $\geq 150$ min./sem.) em idosos de Rio do Sul, SC, 2014 ( $n=213)$.

\begin{tabular}{|c|c|c|c|c|c|c|c|}
\hline \multirow{3}{*}{ Variáveis } & & \multicolumn{6}{|c|}{ Caminhada no deslocamento ( $\geq 150 \mathrm{~min} . / \mathrm{sem}$.) } \\
\hline & & \multirow{2}{*}{$\mathrm{n}$} & \multirow{2}{*}{$\%$} & \multicolumn{2}{|c|}{ Bruta } & \multicolumn{2}{|c|}{ Ajustada $^{a}$} \\
\hline & & & & $\mathrm{OR}$ & IC95\% & OR & IC95\% \\
\hline \multirow[t]{2}{*}{ Presença de academias de ginástica } & Não & 9 & 18,0 & 1 & & 1 & \\
\hline & Sim & 51 & 31,3 & 2,07 & $0,94-4,58$ & 4,16 & $1,72-12,3$ \\
\hline \multirow[t]{2}{*}{ Presença de parques } & Não & 9 & 33,3 & 1 & & 1 & \\
\hline & Sim & 51 & 27,4 & 0,75 & $0,32-1,79$ & 0,62 & $0,16-2,34$ \\
\hline \multirow[t]{2}{*}{ Presença de praças } & Não & 11 & 26,8 & 1 & & 1 & \\
\hline & Sim & 49 & 28,5 & 1,08 & $0,50-2,33$ & 1,13 & $0,32-3,92$ \\
\hline \multirow[t]{2}{*}{ Presença de centro comunitário } & Não & 18 & 33,3 & 1 & & 1 & \\
\hline & Sim & 42 & 26,4 & 0,71 & $0,36-1,39$ & 0,71 & $0,31-1,63$ \\
\hline \multirow[t]{2}{*}{ Presença de academia ao ar livre } & Não & 9 & 25,7 & 1 & & 1 & \\
\hline & Sim & 51 & 28,7 & 1,16 & $0,50-2,64$ & 1,15 & $0,36-3,63$ \\
\hline \multirow[t]{2}{*}{ Presença de igrejas, templos, cultos } & Não & 39 & 33,1 & 1 & & 1 & \\
\hline & Sim & 21 & 22,1 & 0,57 & $0,30-1,06$ & 0,75 & $0,35-1,61$ \\
\hline \multirow[t]{2}{*}{ Presença de calçadas } & Não & 18 & 20,5 & 1 & & 1 & \\
\hline & Sim & 42 & 33,6 & 1,96 & $1,04-3,72$ & 3,50 & $1,48-8,28$ \\
\hline \multirow[t]{2}{*}{ Presença de árvores } & Não & 40 & 28,6 & 1 & & 1 & \\
\hline & Sim & 20 & 27,4 & 0,94 & $0,50-1,77$ & 1,16 & $0,54-2,48$ \\
\hline \multirow[t]{2}{*}{ Trânsito intenso no bairro } & Não & 31 & 28,7 & 1 & & 1 & \\
\hline & Sim & 29 & 27,6 & 0,94 & $0,52-1,72$ & 0,97 & $0,46-2,01$ \\
\hline \multirow[t]{2}{*}{ Existem faixas, sinais e passarelas } & Não & 30 & 24,0 & 1 & & 1 & \\
\hline & Sim & 30 & 34,1 & 1,63 & $0,89-2,99$ & 1,45 & $0,67-3,14$ \\
\hline \multirow[t]{2}{*}{ Existem muitos crimes no bairro } & Não & 49 & 27,8 & 1 & & 1 & \\
\hline & Sim & 11 & 29,7 & 1,09 & $0,50-2,38$ & 1,32 & $0,52-3,33$ \\
\hline \multirow[t]{2}{*}{ É seguro caminhar durante a noite } & Não & 23 & 32,4 & 1 & & 1 & \\
\hline & Sim & 37 & 26,1 & 0,73 & $0,39-1,37$ & 0,84 & $0,36-1,94$ \\
\hline \multirow[t]{2}{*}{ Existência de ruas inclinadas } & Não & 31 & 27,7 & 1 & & 1 & \\
\hline & Sim & 29 & 28,7 & 1,05 & $0,57-1,91$ & 1,15 & $0,56-2,34$ \\
\hline
\end{tabular}

a Ajustada para sexo, faixa etária, escolaridade, estado civil, renda média familiar, estado de saúde e todas as variáveis independentes.

\section{Discussão}

Os resultados do estudo apontam que menos de $30 \%$ dos idosos cumprem as recomendações de caminhada no lazer e no deslocamento ( $\geq 150 \mathrm{~min}$./sem.). Outro importante resultado indica que as características do ambiente associadas com a caminhada diferem em relação ao contexto de lazer e deslocamento. Idosos que percebem a presença de academias ao ar livre próximo da residência possuem menor chance de atingirem níveis recomendados de caminhada no lazer. Por outro lado, àqueles que relataram a existência de ruas inclinadas têm maior chance de serem ativos nesse desfecho.

Já na caminhada no deslocamento perceber academias de ginástica e calçadas nas ruas próximas da residência foram associadas com maior chance de cumprirem as recomendações de caminhada. Os resultados reforçam que além de fatores intrapessoais, como sexo, idade, renda e motivação, características do ambiente do bairro, mesmo em cidades menores do Brasil, estão associados com os níveis de caminhada no lazer e no deslocamento em idosos. Dessa maneira, observou-se que as características do ambiente do bairro estão associadas de modo mais consisten- 
te com a caminhada no deslocamento quando comparada a caminhada no lazer.

A prevalência de idosos que cumprem as recomendações de caminhada no lazer e deslocamento foi baixa. Esses resultados são corroborados por outras evidências. Em São Paulo, a proporção de idosos ativos na caminhada no deslocamento foi mais elevada $(34,8 \%)^{16}$. Já em Curitiba observou-se uma prevalência de $35,0 \%$ de caminhada no lazer ${ }^{22}$. Centros Urbanos, caso de São Paulo e Curitiba, de grande porte apresentam diversidade de locais públicos e privados que favorecem a prática de caminhada no lazer, bem como, de infraestrutura que possibilite o deslocamento ativo da população, diferentemente da cidade de Rio do Sul que é classificada como um município de pequeno porte e que pode apresentar menor número de locais para a caminhada. Nessa perspectiva a proximidade de residências e destinos (espaços de lazer, comércio e serviços) pode ter influenciado nesse achado. Corroborando com essa hipótese, estudo com idosos residentes em 23 estados brasileiros em que o deslocamento foi avaliado por meio do IPAQ quanto a caminhada e uso de bicicleta, constatou que cidades de pequeno porte tendem a não favorecer a caminhada como deslocamento possivelmente devido à proximidade entre residências e locais de serviços e/ou lojas ${ }^{23}$.

Em relação às variáveis ambientais, observou-se que idosos que relataram a presença de academias ao ar livre próximo de sua residência têm menor chance de serem ativos nesse domínio. Resultado contrário foi observado em outros estudos em que a presença de academias no bairro associou-se a recomendação de 150 minutos ou mais de atividade física no lazer em idosos ${ }^{25,26}$. No entanto, estes achados não diferenciam o tipo de academia em análise, bem como, a caminhada não é tratada de forma isolada como atividade de lazer. Isso explica nosso resultado já que a caminhada exige um espaço adequado para sua realização, algo que as academias ao ar livre muitas vezes não dispõem. Corroborando, estudo com 1.796 adultos e idosos norte-americanos constatou que ter acesso a locais para a prática de atividades físicas como parques, clubes e academias nas redondezas de casa ou mesmo no local de trabalho aumentou as chances dos indivíduos realizarem algum tipo de atividade física no lazer ${ }^{27}$. Portanto, a caminhada no lazer é fortemente associada a proximidade de parques ou praças por possibilitar a prática adequada desse tipo de atividade $e^{25,28}$.

Acredita-se que esse tipo de estrutura (academias ao ar livre) não possibilite a prática específica de caminhada no lazer. Outra hipótese refere-se ao fato dos indivíduos com mais idade que costumam caminhar no bairro, durante o tempo livre, e que têm maior confiança na sua capacidade de ser ativo são, talvez, mais propensos a serem críticos da infraestrutura existente para caminhada, com desejo de mais espaços voltados a essa prática, diferentemente, de idosos que não caminham no lazer em sua vizinhança, os quais tendem a assumir essa infraestrutura como suficiente ${ }^{26}$. Estudo de revisão sobre ambiente construído e atividade física envolvendo idosos americanos, constatou que melhorias em instalações e ambientes podem estimular a prática de caminhada dessa população ${ }^{8}$.

Ainda em relação ao lazer, observa-se que idosos que percebem existência de ruas inclinadas têm maior chance de serem ativos na caminhada de lazer. Embora seja em um sentido não esperado a associação pode ser devido ao fato de que entre os idosos que caminham é mais perceptível à visualização de locais íngremes, que dificultam a caminhada, diferente daqueles que não caminham, assim o declive e aclive nas ruas é menos percebido por estes idosos. Ademais, embora os idosos percebam esse aspecto como restritivo à atividade física, os mesmos não deixam de realizá-la, pois idosos ativos se expõem mais frequentemente a condições ad- 
versas $^{12}$. Aliás, a cidade de Rio do Sul está situada numa área geográfica de muitos morros, nos quais a população buscou construir suas residências, já que a região baixa do município é constantemente atingida por enchentes ${ }^{24}$.

Quanto à caminhada no deslocamento, evidenciou-se que a presença de academias de ginástica foi associada a maior chance de idosos atingirem as recomendações de caminhada. Acredita-se que devido o presente estudo avaliar essa estrutura de maneira individual, diferentemente de outras pesquisas, ao não incluir um conjunto de outras variáveis relacionadas a locais e serviços que podem estar relacionadas com facilitadores para a prática de caminhada em idosos. Neste sentido, o resultado é um indicativo de que a proximidade a estruturas comerciais e de serviços, como academias de ginástica, podem estimular o deslocamento ativo dessa população ${ }^{16}$. Estudos indicam que ambientes urbanos com diversidade de locais/destinos são fatores importantes para esse tipo de atividade física pela população idosa, já que estes preferem caminhar a destinos mais próximos de sua residência ${ }^{10,11,26,29}$. Winters et al. ${ }^{10}$ ao estudarem 1.309 idosos canadenses quanto ao Street Smart Walk Score e a percepção de caminhada, concluíram que bairros com maior uso misto do solo, ou seja, diversidade de comércio e de instalações de lazer podem contribuir para que idosos atinjam as recomendações de atividade física, já que a maioria dos deslocamentos realizados por meio da caminhada fazem parte do cotidiano.

Nessa perspectiva, outro achado relevante foi à associação entre a existência de calçadas e caminhada no deslocamento. Esse resultado é corroborado por outras evidências nacionais ${ }^{13,14}$ e internacionais ${ }^{10,30}$. Estudos de revisão sistemática e meta-análise demonstraram fortes evidências quanto a associação entre a presença de calçadas em boas condições e a caminhada no deslocamento ${ }^{8,30}$. Isso mostra que esse tipo de estrutura é fundamental para um comportamento ativo quanto à caminhada no deslocamento em idosos, independentemente da densidade populacional do município ${ }^{14}$.

Essas associações dos fatores ambientais e lazer alertam para uma possível influência do contexto urbano quanto à presença inadequada (academias ao ar livre e ruas inclinadas) ou insuficiente de estrutura destinada à prática de caminhada no lazer em município com menor porte populacional. Considerando, que na maioria das vezes as cidades de pequeno porte carecem de infraestrutura voltada à atividade física, é necessário há diversidade de destinos e presença de calçadas adequadas, sendo este um fator preponderante para o aumento dos níveis mínimos de atividade física da população idosa. Destaca-se ainda que a caminhada é utilizada como um dos poucos recursos disponíveis para manutenção da saúde do idoso, por ser potencialmente mais acessível ${ }^{8,16}$, de baixo impacto e com elevados níveis de aderência ${ }^{6,7}$. Tendo em vista que este é um dos primeiros estudos envolvendo características ambientais e a caminhada no lazer e no deslocamento, de uma cidade de pequeno porte no Brasil, recomenda-se a realização de estudos futuros sobre auditagem de calçadas e levantamento do tipo e qualidade das estruturas voltadas a atividade física nesse contexto.

Estes resultados fornecem subsídios importantes para tomada de decisão dos gestores públicos, com base em determinadas características do ambiente do bairro como a presença de calçadas e estruturas adequadas a prática de atividade física, especialmente a caminhada. Para tanto, o planejamento urbano deve envolver não apenas a construção desses espaços, mas assim como a manutenção e a fiscalização permitindo sua utilização. Maior uso misto do solo, entendido como a maior integração de áreas residenciais, comerciais e de lazer, pode promover maior 
número de deslocamentos e por outro lado diminuir o deslocamento inativo. Para tanto, a presença de calçadas parece ser o fator primordial para que o município otimize a caminhada pela comunidade idosa.

A análise e interpretação dos resultados deste estudo apresentam algumas limitações. O delineamento transversal não permite estabelecer relação de causa e efeito entre exposição e desfecho. Segundo, a percepção individual do ambiente pode possuir viés de memória, ou seja, o idoso não lembrar de determinadas características da vizinhança do seu bairro, porém utilizou-se um instrumento padronizado e amplamente empregado para esse fim. Terceiro, a aplicação do IPAQ fornecendo uma medida indireta da prática de caminhada no lazer e deslocamento, embora seja um instrumento validado para avaliação de atividade física em idosos brasileiros ${ }^{20}$. Quarto, o fato da amostra ter sido obtida por conveniência o que não permite a generalização dos resultados, bem como, o fato de não poder estratificar as análises segundo o sexo, a idade e o nível socioeconômico.

Entre os pontos positivos está o contexto em que o estudo foi conduzido, sendo um dos primeiros trabalhos há investigar as características ambientais e a caminhada no lazer e no deslocamento em idosos residentes em cidade de pequeno porte. Também, os resultados permitem identificar pontos importantes e contundentes quanto a percepção do ambiente em idosos integrantes de grupos de convivência do município, dados esses, fundamentais para a elaboração de novas ações para oxigenar a atividade física nessa população.

Conclui-se que é baixa a prevalência de idosos que cumprem a recomendação de caminhada no lazer e deslocamento, tendo uma relação com a falta de estrutura adequada a prática desse tipo de atividade, principalmente no lazer. É necessário maior uso misto do solo (diversidade de áreas residenciais, comerciais e lazer) com políticas públicas que facilitem o acesso mediante a presença de calçadas e de infraestrutura voltada à caminhada no lazer e deslocamento para contribuir no aumento do comportamento ativo na vizinhança próxima da residência de idosos moradores em cidade de menor densidade populacional.

\section{Agradecimentos}

Os autores agradecem ao apoio recebido pela UNIDAVI através do FAPE (Fundo de Apoio à Pesquisa e Extensão), pois esta publicação é resultante do Projeto "atividade física e percepção do ambiente em idosos residentes em Rio do Sul.

\section{Contribuiç̧ões dos autores}

Balbé G.P. (0000-0002-5570-0667) e Rech C.R. (0000-0002-9647-3448) participaram da concepção do estudo, análise, interpretação dos dados e redação final do artigo. Wathier A.C. (0000-0003-2886-5420) participou da concepção e revisão do artigo. Os autores declaram que não há nenhum conflito de interesse.

\section{Referências}

1. Sallis JF, Cerin E, Conway TL, Adams MA, Frank LD, Pratt M, et al. Physical activity in relation to urban environments in 14 cities worldwide: a cross-sectional study. Lancet. 2016;387(10034):2207-17.

2. Silva ICMD, Mielke GI, Nunes BP, Böhm AW, Blanke ADO, Nachtigall MDS, et al. Espaços públicos de lazer: distribuição, qualidade e adequação à prática de atividade física. Rev Bras Ativ Fís Saúde. 2015;20(1):82-92.

3. Kohl HW 3rd, Craig CL, Lambert EV, Inoue S, Alkandari JR, Leetongin G, et al. The pandemic of physical inactivity: global action for public health. Lancet. 2012;380:294-305. 
4. Bauman AE, Reis RS, Sallis JF, Wells JC, Loos RJ, Martin BW, et al. Correlates of physical activity: why are some people physically active and others not? Lancet. 2012;380(9838):258-71.

5. Wang Y, Chau CK, Ng WY, Leung TM. A review on the effects of physical built environment attributes on enhancing walking and cycling activity levels within residential neighborhoods. Cities. 2016;50:1-15.

6. Hanson $\mathrm{S}$, Jones A. Is there evidence that walking groups have health benefits? a systematic review and meta-analysis. Br J Sports Med. 2015;0:1-7.

7. Costa TB, Ribeiro LHM, Neri AL. Prevalence of and factors associated with leisure-time physical activity in older adults from seven Brazilian cities: data from the FIBRA study. Rev Bras Ativ Fis Saúde. 2015;20(2):174-183.

8. Haselwandter EM, Corcoran MP, Folta SC, Hyatt R, Fenton M, Nelson ME. The built environment, physical activity and aging in the united states: a state of the science review. J Aging Phys Act. 2015;23:323-29.

9. Murtagh EM, Nichols L, Mohammed MA, Holder R, Nevill AM, Murphy MH. The effect of walking on risk factors for cardiovascular disease: an updated systematic review and meta-analysis of randomised control trials. Prev Med. 2015;72:34-43.

10. Winters M, Barnes R, Venners S, Ste-Marie N, McKay H, Sims-Gould J, et al. Older adults' outdoor walking and the built environment: does income matter? BMC Public Health. 2015;15(876):1-8.

11. Winters M, Voss C, Ashe MC, Gutteridge K, McKay H, Sims-Gould J. Where do they go and how do they get there? older adults' travel behaviour in a highly walkable environment. Soc Sci Med. 2015;133:304-12.

12. Giehl MWC, Schneider IJC, Corseuil HX, Benedetti TRB, d'Orsi E. Atividade física e percepção do ambiente em idosos: estudo populacional em Florianópolis. Rev Saúde Públ. 2012;46(3):516-25.

13. Giehl MWC, Hallal PC, Brownson RC, d'Orsi E. Exploring associations between perceived measures of the environment and walking among Brazilian older adults. J Aging Health. In press, 2016.

14. Giehl MWC, Hallal PC, Corseuil CW, Schneider IJ, d'Orsi E. Built environment and walking behavior among Brazilian older adults: a population-based study. J Phys Act Health. 2016;13(6):617-24.

15. Salvador EP, Reis RS, Florindo AA. Practice of walking and its association with perceived environment among elderly Brazilians living in a region of low socioeconomic level. Int J Behav Nutr Phys Act. 2010;7(67):1-7.

16. Salvador EP, Reis RS, Florindo AA. A prática de caminhada como forma de deslocamento e sua associação com a percepção do ambiente em idosos. Rev Bras Ativ Fís Saúde. 2009;14(3):197-205.

17. IBGE. Instituto Brasileiro de Geografia e Estatística. Censo demográfico 2010. [citado 2016 jun 15]. Disponível em: 〈www.cidades.ibge.gov.br〉.

18. PNUD. Programa das Nações Unidas para o desenvolvimento. Atlas do desenvolvimento humano no Brasil, 2013. [citado 2016 fev 27]. Disponível em: 〈http://www.pnud.org.br/atlas/>.

19. Faul F, Erdfelder E, Lang A-G, Buchner A. G*Power 3: a flexible statistical power analysis program for the social, behavioral, and biomedical sciences. Behav Res Methods. 2007;39(2):175-91.

20. Benedetti TRB, Mazo GZ, Barros MVG. Aplicação do Questionário Internacional de Atividades Físicas para avaliação do nível de atividades físicas de mulheres idosas: validade concorrente e reprodutibilidade teste re-teste. R Bras Ci Mov. 2004;12(1):25-34.

21. Malavasi LDM, Duarte MDFDS, Both J, Reis RS. Escala de Mobilidade Ativa no Ambiente Comunitário News Brasil: tradução e reprodutibilidade. Rev Bras Cineantropom Desempenho Hum. 2007;9(4):339-50.

22. Parra DC, Hoehner CM, Hallal PC, Ribeiro IC, Reis R, Brownson RC, et al. Perceived environmental correlates of physical activity for leisure and transportation in Curitiba, Brazil. Prev Med. 2011;52(3-4):234-38.

23. Madeira MC, Siqueira FCV, Facchini LA, Silveira DSd, Tomasi E, Thumé E, et al. Atividade física no deslocamento em adultos e idosos do Brasil: prevalências e fatores associados. Cad Saúde Pública. 2013;29:165-74.

24. Espíndola MA, Nodari ES. Enchentes inesperadas? vulnerabilidades e políticas públicas em Rio do Sul - SC, Brasil. Rev Esboços. 2103;20(30):9-34. 
25. Costa BGG, Freitas CDLR, Silva KS. Atividade física e uso de equipamentos entre usuários de duas Academias ao Ar Livre. Rev Bras Ativ Fis Saúde. 2016;21(1):29-38.

26. Carlson JA, Sallis JF, Conway TL, Saelens BE, Frank LD, Kerr J, et al. Interactions between psychosocial and built environment factors in explaining older adults' physical activity. Prev Med. 2012;54(1):68-73.

27. Huston SL, Evenson KR, Bors P, Gizlice Z. Neighborhood environment, access to places for activity, and leisure-time physical activity in a diverse North Carolina population. Am J Health Promot. 2003;18(1):58-69.

28. Sugiyama T, Francis J, Middleton NJ, Owen N, Giles-Corti B. Associations between recreational walking and attractiveness, size, and proximity of neighborhood open spaces. Am J Public Health. 2010;100(9):1752-57.

29. Perchoux C, Kestens Y, Brondeel R, Chaix B. Accounting for the daily locations visited in the study of the built environment correlates of recreational walking (the RECORD Cohort Study). Prev Med. 2015;81:142-9.

30. Ding D, Adams MA, Sallis JF, Norman GJ, Hovell MF, Chambers CD, et al. Perceived neighborhood environment and physical activity in 11 countries: do associations differ by country? Int J Behav Nutr Phys Act. 2013;10(57): 1-11.

Endereço para

Correspondência

Giovane Pereira Balbé

gbalbe@unidavi.edu.com.br
Rua Doutor Guilherme Gemballa, no 13 ,

Jardim América, CEP: 89160-932

Rio do Sul, Santa Catarina, Brasil.

Telefone: (47) 35316015
RECEBIDO 30/08/2016

REVISADO 21/12/2016

APROVADO $\quad 27 / 12 / 2016$ 